\title{
IL TEISMO FILOSOFICO DI ALASDAIR MACINTYRE A CONFRONTO CON LA TEOLOGIA POST-LIBERALE E POST-MODERNA
}

\author{
Alasdair Maclntyre's philosophical theism in conversation with postliberal and \\ postmodern theologies
}

Carlo Leonardi

Istituto Superiore di Scienze Religiose «Beato Gregorio X» (Arezzo)

\begin{abstract}
Sommario: Il presente saggio si propone di delineare I'"habitus teologico", che caratterizza il modus philosophandi di Maclntyre: habitus che si evince - a latere del capolavoro macintyriano, After Virtue (1981) - in numerose opere preparatorie, coeve o anche successive, come ad es. The Logical Status of Religious Belief (1957), Difficulties in Christian Belief (1959), The Debate about God (1969), Can Medicine Dispense with a Theological Perspective on Human Nature? (1977), Theology, Ethics, and the Ethics of Medicine and Health Care (1979), Dr. Küng's Fiasco (1981), Which God Ought We to Obey and Why? (1986), What Has Christianity to Say to the Moral Philosopher (2006), God, Philosophy, Universities (2009), On Being a Theistic Philosopher in a Secularized Culture (2010), ecc., le quali costituiscono, a mio avviso, un essenziale corredo dell'etica delle virtù promossa dal filosofo scozzese. Pertanto, non desta sorpresa che Maclntyre sia stato spesso coinvolto in dibattiti riguardanti l'etica teologica e la teologia naturale; sennonché il suo approccio innovativo ha indotto taluni interpreti a istituire una sorta di parallelo tra il teismo filosofico macintyriano e alcune inedite correnti teologiche del Novecento: la teologia narrativa postliberale, la "radical orthodoxy", ecc. Sebbene Maclntyre si sia astenuto dal pronunciarsi in merito a questi tentativi di "appropriazione teologica" del suo pensiero, probabilmente maggiori analogie possono essere colte con quanti, nel secolo scorso, hanno proseguito la ricerca filosofica e teologica nell'alveo della tradizione aristotelico-tomista, a cui oggi afferisce de iure et de facto anche l'affluente del "tomismo analitico".
\end{abstract}

Parole Chiavi: Alasdair MacIntyre, teismo filosofico, tomismo analitico, teologia post-liberal

\begin{abstract}
This paper aims at sketching what is herein called the "theological habitus", which characterizes Maclntyre's modus philosophandi, even if it is not as plainly evident in his masterpiece, After Virtue (1981), as in many preparatory, collateral and successive works like The Logical Status of Religious Belief (1957), Difficulties in Christian Belief (1959), The Debate about God (1969), Can Medicine Dispense with a Theological Perspective on Human Nature? (1977), Theology, Ethics, and the Ethics of Medicine and Health Care (1979), Dr. Küng's Fiasco (1981), Which God Ought We to Obey and Why? (1986), What Has Christianity to Say to the Moral Philosopher (2006), God, Philosophy, Universities (2009), On Being a Theistic Philosopher in a Secularized Culture (2010), ecc. Thus, such an habitus has naturally involved Maclntyre in academic debates regarding theological ethics and philosophical theism; but his innovative approach has also induced many interpreters to link Maclntyre's thought to twentieth century new theological movements: postliberal narrative theology, radical orthodoxy, ecc. Although Maclntyre has never stated a comment on these kinds of "theological appropriation" of his oeuvre, probably major similarities can be found with those who, in the meantime, have prosecuted philosophical and theological research within Aristotelian-Thomistic tradition, which today includes de jure et de facto "analytical Thomism".
\end{abstract}

Keywords: Alasdair MacIntyre, philosophical theism, analytical Thomism, postliberal theology 
Nel breve spazio del presente lavoro, intendo dar conto dell' "habitus teologico" che caratterizza lo stile filosofico di Alasdair Maclntyre 1 : "religiously musical" è infatti — a mio avviso - il più insolito, e allo stesso tempo il più suadente, epiteto attribuito al filosofo scozzese dai teologi James Gustafson e Stanley Hauerwas ${ }^{2}$. Tale habitus è altresì esaltato dalla diffusa propensione a saldare insieme - senza apparente soluzione di continuità - il modus philosophandi macintyriano e alcune recenti figure della teologia cristiana post-liberale e post-moderna, di cui ci occuperemo in seguito: soprattutto la "teologia narrativa" di Hans Frei, George Lindbeck, et. al., ma anche la "radical orthodoxy" di John Milbank, Catherine Pickstock, Graham Ward, et. al. ${ }^{3}$ Sebbene Maclntyre non abbia replicato direttamente a simili tentativi di "appropriazione teologica" del suo pensiero, ritengo, comunque, che i tempi siano maturi per riconoscere - a lui e al "tomismo analitico" - un ruolo sui generis nell'evoluzione della tradizione aristotelico-tomista del XX secolo ${ }^{4}$ : ruolo distinto, ma non separato, rispetto a quello coevo di quanti - in ambito continentale - hanno tenacemente proseguito la ricerca filosofica e teologica nel solco di Tommaso, come ad esempio Marie-Dominique Chenu, Yves Congar, Cornelio Fabro, Etienne Gilson, Jacques Maritain, Edith Stein, et al.

\section{Etica filosofica e teismo cristiano nell'opera di Maclntyre}

\footnotetext{
${ }^{1}$ Per una trattazione più ampia e articolata del pensiero di Maclntyre, quale filosofo morale e teologo razionale, rinvio al mio saggio monografico: Alasdair Maclntyre. Sul crocevia tra etica, filosofia della religione e teologia, Aracne, Roma 2013.

2 J. GUSTAFSON - S. HAUERWAS, Editorial, in "The Journal of Medicine and Philosophy», 4 (1979), pp. 345-6 (spec. p. 346). Se si valuta per intero l'originale itinerarium mentis in deum intrapreso dal filosofo scozzese, si possono effettivamente apprezzare le parole di B.J. SHANLEY (The Thomist Tradition, Kluwer, Dordrecht 2002, p. 149): "Maclntyre's moral theory is by far the most theocentric offered by any prominent Thomist».

${ }^{3}$ Cfr. ad es. D. Fergusson (Community, Liberalism and Christian Ethics, Cambridge U.P., Cambridge 1998, p.5), il quale sostiene che nell'opera di Hauerwas si trovi «il più prossimo "analogo teologico" della filosofia macintyriana»; oppure la lettura sinottica di Maclntyre e Lindbeck offerta da D. TRENERY (Alasdair Macintyre, George Lindbeck, and the Nature of Tradition, Pickwick, Eugene 2014); oppure ancora l'accostamento tra Maclntyre e la "radical orthodoxy" promosso da T. RowLAND (Culture and the Thomist Tradition after Vatican II, Routledge, London-New York 2003).

${ }^{4}$ Sulla rinascita della teologia filosofica in ambito analitico, cfr. invece J.F. HARRIS, Analytic Philosophy of Religion, Kluwer, Dordrecht 2002; O.D. CRISP - M.C. REA (eds.), Analytic Theology. New Essays in the Philosophy of Theology, Oxford U.P., Oxford 2009; M. MICHELETTI, La teologia razionale nella filosofia analitica, Carocci, Roma 2010, ecc.
} 
Ciò detto, è noto che fin dalla prima edizione del 1981, ovvero da più di un quarto di secolo oramai, l'opus magnum di Maclntyre, After Virtue. A Study in Moral Theory, ha catalizzato l'attenzione dei maggiori interpreti del pensiero del filosofo scozzese: quanto egli ha scritto a latere di After Virtue - specie nell'ambito della filosofia della religione e della teologia naturale: in veste di coeditore insieme a Antony Flew, New Essays in Philosophical Theology (1955), Stephen Toulmin e Ronald Hepburn, Metaphysical Beliefs (1957), Paul Ricoeur, The Religious Significance of Atheism (1969), e, in prima persona, Difficulties in Christian Belief (1959), Marxism and Christianity (1968), Which God Ought We to Obey and Why? (1986), What Has Christianity to Say to the Moral Philosopher (2006), God, Philosophy, Universities (2009), On Being a Theistic Philosopher in a Secularized Culture (2010), ecc. - è stato inevitabilmente eclissato dall'enorme successo ottenuto dal capolavoro macintyriano.

Quest'esito non risulta affatto irragionevole, né tanto meno sorprendente, qualora si prenda in esame l' "indole genealogico-sovversiva” che pervade After Virtue, laddove i canoni meta-etici - a lungo imperanti nelle università anglosassoni - sono stati energicamente confutati e infine rigettati da Maclntyre, alla luce di un fecondo dialogo instaurato tra la "filosofia analitica" e una "versione aggiornata" dell'etica aristotelico(-tomista)

Un simile dialogo era stato preconizzato, negli anni Cinquanta e Sessanta del secolo scorso, da alcuni studi pioneristici compiuti da allievi e colleghi di Wittgenstein: G.E.M. Anscombe, Peter Geach, Georg Henrik von Wright, et. al. Ciononostante, After Virtue e le successive opere - che compongono insieme la più nota trilogia di Maclntyre: Whose Justice? Which Rationality? (1988) e Three Rival Versions of Moral Enquiry (1990) - hanno promosso con un impeto senza pari tale approccio all'etica e all'antropologia filosofica, in principio etichettato come "tomismo wittgensteiniano" e, successivamente, 
applicato anche alla filosofia della mente, alla metafisica e all'ontologia, "tomismo analitico" ${ }^{\prime 5}$.

After Virtue costituisce, dunque, un "punto di non ritorno" nella vicenda umana e intellettuale di Maclntyre ${ }^{6}$, a causa della rinnovata enfasi posta sul paradigma dell'etica delle virtù quale tertia via al di là della deontologia e dell'utilitarismo ${ }^{7}$. Allo stesso tempo, però, condivido l'opinione secondo cui ciò che è rimasto largamente implicito in After Virtue è in quale misura «Dio - il Dio del teismo filosofico - entri inesorabilmente nella teoria morale di Maclntyre e nella sua descrizione della vita buona, poiché è indubbio che egli abbia combattuto nel corso di tutta la vita con la questione di quale posto possa avere Dio all'interno di una teoria morale che sia razionalmente difendibile ${ }^{8}$.

Chiunque voglia ripercorrere, in sede storiografica, l'evoluzione complessiva del pensiero di Maclntyre, dovrà, quindi, necessariamente cimentarsi con le opere in cui il

\footnotetext{
${ }^{5}$ Non posso soffermarmi oltre sul significato e sulla pertinenza di siffatte classificazioni, né sulla loro congruenza in relazione a Maclntyre. Credo tuttavia che, entro i limiti propri di qualunque tassonomia, vi siamo numerosi indizi che permettono di ascrivere il filosofo scozzese tra i maggiori fautori del tomismo analitico, non ultimo il fatto che l'espressione in questione sia stata utilizzata per la prima volta da Haldane nelle lectures tenute alla Notre Dame University su invito dello stesso Maclntyre nel 1992; cfr. J. HALDANE, Analytical Thomism, in T. HONDERICH (ed.), The Oxford Companion to Philosophy, Oxford U.P., Oxford 2005 ${ }^{2}$ p. 918: "The expression "analytical Thomism" is rarely employed, but it usefully identifies aspects of the writings of philosophers such as Anscombe, Donagan, Geach, Grisez, Kenny, and Maclntyre». In merito, rinvio altresì al numero monografico curato da J. HALDANE, Analytical Thomism, in "The Monist», 80 (1997) 4; ID. (ed.), Mind, Metaphysics, and Value in the Thomistic and Analytical Traditions, University of Notre Dame Press, Notre Dame 2002; C. PATERSON - M.S. PUGH (eds.), Analytical Thomism: Traditions in Dialogue Ashgate, Aldershot 2006; M. MiCheletTI, Tomismo analitico, Morcelliana, Brescia 2007; F. KERR, Un thomisme analytique?, in «Revue des Sciences Philosophiques et Théologiques», 92 (2008), pp. 557-67; G. VENTIMIGLIA, To be o esse? La questione dell'essere nel tomismo analitico, Carocci, Roma 2012, ecc.

${ }^{6}$ Per una recente nota biografica sull'Autore, cfr. C.S. Lutz, Alasdair Chalmers Maclntyre (1929- ), in «Internet Encyclopedia of Philosophy», http://www.iep.utm.edu/mac-over/.

7 Sull'etica delle virtù, nelle sue molteplici declinazioni, e sul primato che Maclntyre attribuisce alla tradizione aristotelico-tomista esiste oramai una vasta e incessante letteratura. Per alcune recenti introduzioni ai temi maggiormente dibattuti nell'ambito dell'etica delle virtù, rinvio a D.C. RUSSELL, The Cambridge Companion to Virtue Ethics, Cambridge U.P., Cambridge 2013; S. van Hooft (ed.), The Handbook of Virtue Ethics, Routledge, London-New York 2014 e L. BESSER-JONES - M. SLOTE (eds.), The Routledge Companion to Virtue Ethics, Routledge, London-New York 2015. Ha altresì criticato una certa "normalizzazione" dell'etica delle virtù, rispetto alle posizioni radicali della Anscombe e di Maclntyre, T. BREWER (The Retrieval of Ethics, Oxford U.P., Oxford 2011, pp. 3-4), mentre ha illustrato efficacemente la loro filiazione all'interno del tomismo analitico, M. MICHELETTI (II problema dell'etica nel tomismo analitico, in corso di pubblicazione in Atti del Convegno sul Tomismo creativo. Letture contemporanee del Doctor Communis, Bologna, 3-4 Dicembre 2013).

8 T.D. D'ANDREA, Tradition, Rationality, and Virtue: The Thought of Alasdair Maclntyre, Ashgate, Aldershot 2006, p. 389.
} 
filosofo scozzese indaga il rapporto tra l'etica filosofica e la teologia morale, tra i moderni miti del progresso e l'escatologia cristiana, tra l'attuale crisi morale e l'abbandono del teismo classico, tra la secolarizzazione incipiente e la possibilità residua di poter vivere un cristianesimo autentico, all'interno di una società concepita etsi deus non daretur, ecc.: opere che non costituiscono certamente "semplici appendici", composte in tono minore, bensì "essenziali complementi" dell'etica delle virtù macintyriana. Da esse, infatti, emerge come Maclntyre, parallelamente alla stesura di After Virtue, abbia preso parte attiva a un dibattito assai vivace tra teologi morali: James Gustafson, Stanley Hauerwas, Richard McCormick, Paul Ramsey, et al.; e studiosi di etica filosofica: Antony Flew, William Frankena, Basil Mitchell, et al., i quali si interrogavano sui caratteri distintivi dell'etica teologica (Christian ethics), alla luce di una legittima pluralità e diversità di tradizioni ebraico-cristiane ${ }^{9}$.

Al centro del confronto, vi erano i concetti costitutivi di qualsiasi percorso di etica teologica: "narrazione biblica", "mito", "legge naturale" e "comandamenti divini", "virtù cardinali e teologali", "storia umana" e "storia della salvezza", "escatologia", ecc. La discussione rischiava, però, - a giudizio di Maclntyre - di risultare demodé, o peggio senseless, da un lato, a causa delle teorie metaetiche che tendevano ad attribuire un mero carattere espressivo ai giudizi etici, privandoli di qualunque valore cognitivo; e, dall'altro, a causa del processo storico e sociale di secolarizzazione che mutava

\footnotetext{
${ }^{9}$ Per i necessari approfondimenti sul dibattito in questione, rinvio a C. LEONARDI, Alasdair Maclntyre, cit., pp. 121-41. Cfr. altresì i seguenti riferimenti bibliografici: in campo filosofico, A. MACINTYRE, Can Medicine Dispense with a Theological Perspective on Human Nature?, in D. CALlaHAN - H.T. ENGELHARDt JR. (eds.), Knowledge, Value and Belief, The Hastings Center, New York 1977, pp. 25-43; ID., Theology, Ethics, and the Ethics of Medicine and Health Care: Comments on Papers by Novak, Mouw, Roach, Cahill, and Hartt, in "The Journal of Medicine and Philosophy», 4 (1979), pp. 435-43; B. MITCHELL, Morality: Religious and Secular, Clarendon, Oxford 1980; K. NIELSEN, God and the Basis of Morality, in "The Journal of Religious Ethics», 10 (1982), pp. 335-50; W. FRANKENA, The Potential of Theology for Ethics, in E.E. SHeLP (ed.), Theology and Bioethics, Reidel, Dordrecht 1985, pp. 49-64, ecc.; in campo teologico, P. RAMSEY, Kant's Moral Theology or a Religious Ethics?, in D. CALLAHAN - H.T. ENGELHARDT JR. (eds.), Knowledge, Value and Belief, cit., pp. 44-74; C. CURRAN - R. McCORMICK (eds.), The Distinctiveness of Christian Ethics: Readings in Moral Theology No. 2, Paulist, New York 1980; J. GUSTAFSON, Ethics from a Theocentric Perspective, 2 Vols., The University of Chicago Press, Chicago 1981-1984; S. HAUERWAS, On Keeping Theological Ethics Theological, in S. HAUERWAS - A. MAcINTYRE (eds.), Revisions: Changing Perspective in Moral Philosophy, University of Notre Dame Press, Notre Dame 1983, pp. 16-42, ecc.
} 
profondamente il contesto di riferimento in cui i concetti propri dell'etica teologica potevano trovare significato e giustificazione.

Maclntyre appariva, quindi, determinato a confutare la fallacia delle teorie metaetiche, ma, parimenti, riteneva di non poter non constatare una certa atrofia e deterioramento di quegli stessi concetti, divelti oramai da un comune quadro etico e sociale di riferimento. In altre parole, la secolarizzazione - agli occhi del filosofo scozzese - faceva esplodere le aporie interne al teismo cristiano, le quali non avevano impedito la fede nel Medioevo, ma oggi apparivano ai più inconciliabili con la ratio (postImoderna: egli, tuttavia, malgrado i rilievi mossigli da alcuni critici che hanno stigmatizzato un suo presunto "tomismo postmoderno" ${ }^{10}$, ha costantemente respinto tutte le metamorfosi della filosofia postmoderna (o post-filosofia) di Richard Rorty, et al. ${ }^{11}$, così come le molteplici varianti di "teologia della secolarizzazione", promosse da John Robinson, Paul Tillich, Paul van Buren, et al. ${ }^{12}$

\section{Le insanabili aporie della "teologia della secolarizzazione"}

In ossequio all'impegno di ricostruire, nell'ambito della pratica filosofica, il legame reciso con le diverse scienze umane - da cui scaturisce l'interesse di Maclntyre per le scienze storico-sociali, prima ancora che per quelle biblico-teologiche - si può asserire che, alla radice dei rari, ancorché rivelatori, sondaggi teologici macintyriani, vi sia

\footnotetext{
${ }^{10}$ Cfr. T.S. HIBBS, Maclntyre's Postmodern Thomism: Reflections on Three Rival Versions of Moral Enquiry, in «The Thomist», 57 (1993), pp. 277-97.

${ }^{11}$ La ricezione - da parte di MacIntyre - dell'opera di Rorty e di altri alfieri della postmodernità non è stata certo simpatetica: cfr. ID., Reviews of Richard Rorty, Philosophy and the Mirror of Nature; Stanley Cavell, The Claim of Reason: Wittgenstein, Skepticism, Morality and Tragedy; Ted Honderich - Myles Burnyeat (eds.), Philosophy as It Is, in «London Review of Books», 11 (1980), pp. 15-6; ID., Philosophy, the "Other" Disciplines, and Their Histories: A Rejoinder to Richard Rorty, in «Soundings", 65 (1982), pp. 12745; ID., Philosophy: Past Conflict and Future Direction, in «Proceedings and Addresses of the American Philosophical Association», 61 (1987), pp. 81-7 e ID., Review of Richard Rorty, Contingency, Irony, and Solidarity, in «The Journal of Philosophy», 87 (1990), pp. 708-11.

${ }^{12}$ Cfr. A. MACINTYRE, God and the Theologians. Review of John Robinson, Honest to God, in «Encounter», 21 (1963), pp. 3-10 (ora in ID., Against the Self-Images of the Age: Essays on Ideology and Philosophy, 1971, Duckworth, London 19832, pp. 12-26), dove l'Autore rileva che, a partire dal "processo di demitologizzazione" di Rudolf Bultmann, il cristianesimo è stato secolarizzato in una sorta di "esistenzialismo teistico" heideggeriano, che «diviene potenzialmente indipendente dall'occorrenza di particolari eventi, verificatisi nella Palestina del primo secolo, e persino dall'esistenza di una divinità soprannaturale» (spec. p. 16).
} 
l'intenzione di far uscire la teologia dal vicolo cieco descritto a chiare lettere dal sociologo Peter Berger:

Se si vuol avere una parte importante nella società moderna, allora bisogna ampiamente rinunciare ai contenuti religiosi tradizionali [secolarizzazione], proprio perché essi sono irrilevanti nella situazione odierna. Se invece si vuole continuare ad affermare tali contenuti come realtà, allora bisogna abituarsi alle strutture sociali dell'esistenza di setta [...]. Tertium non datur ${ }^{13}$.

È con tale dilemma che si sono misurati i più acuti teologi del Novecento - in particolare, i cosiddetti "teologi della secolarizzazione", John Robinson, Paul Tillich, Paul van Buren, et al., ma anche i fautori della "teologia della speranza", vedi Jürgen Moltmann, della "teologia politica", Johannes Baptist Metz in primis, della "teologia narrativa", ovvero Hans Frei, Stanley Hauerwas, George Lindbeck, et al., e della "radical orthodoxy", ossia John Milbank, Catherine Pickstock, Graham Ward, et. al. - e probabilmente esso manca tuttora di una risoluzione pienamente soddisfacente ${ }^{14}$.

Ebbene, Maclntyre, da parte sua, ha manifestato un irremovibile dissenso nei confronti della teologia della secolarizzazione, attraverso la pubblicazione di alcuni saggi e recensioni: God and the Theologians. Review of John Robinson, Honest to God (1963), Secularization and Moral Change (1967), The Debate about God (1969) e Dr. Küng's Fiasco. Review of Hans Küng, Does God Exist? (1981), in cui egli individua il nucleo teorico di tale scuola teologica nella volontà di

preservare il "nocciolo" del cristianesimo, gettandone via la "scorza" invecchiata, [... vale a dire nella volontà di affrontare la moderna crisi del teismo] non attraverso il rifiuto tout court della religione (B. Russell), né ritirandosi nell'ambito conchiuso di una forma di vita e di pensiero religiosi ( $K$. Barth), né attaccando la cultura secolare in nome della superiorità di una forma di arcaismo consapevolmente assunto (T.S. Eliot). [... I teologi della secolarizzazione] possono non essere d'accordo riguardo ai criteri con i quali separare il nocciolo dalla scorza, ma sono unanimi nel ritenere che un moderno

13 P. Berger, Contributo alla sociologia di minoranze conoscitive, in Dialogo: sociologia e religione, Morcelliana, Brescia 1969, pp. 57-66 (spec. p. 65).

${ }^{14}$ Per una pregevole introduzione alle principali correnti teologiche del Novecento, cfr. D. FORD - R. MUERS (eds.), The Modern Theologians: An Introduction to Christian Theology since 1918, Blackwell, Malden $2005^{3}$. 
punto di vista secolare si accordi pienamente con la sostanza della fede cristiana $^{15}$.

Quella appena illustrata sembra essere, altresì, l'impostazione teologica prediletta dal "pensiero debole" di Gianni Vattimo, e di altri filosofi postmoderni, per i quali la secolarizzazione, il disincanto del mondo moderno, costituirebbero, in realtà, l'inveramento del cristianesimo: "Secolarizzazione non è un termine che contrasti con l'essenza del messaggio [cristiano], ma ne è un aspetto a sua volta costitutivo: come evento salvifico ed ermeneutico, l'incarnazione di Gesù (la kénōsis, l'abbassamento di Dio) è essa stessa, anzitutto, un fatto archetipo di secolarizzazione» ${ }^{16}$.

MacIntyre ravvisa in simili affermazioni una sorta di "ateismo teologico", ovvero il tentativo di continuare ad usare il linguaggio religioso per mascherare, in verità, una completa decostruzione e uno svuotamento dall'interno del teismo classico, dissolto in un "vacuum ateistico" 17 :

\begin{abstract}
Tillich, ad esempio, impiega il tradizionale linguaggio teistico - ovvero la caratterizzazione di Dio come "il fondamento dell'essere" - accanto ad una sorta di "metafisica esistenziale", che fornisce la cornice nella quale situare siffatto vocabolario. Quando si tratta di spiegare il proprio concetto di Dio, egli lo identifica con ciò che costituisce l' "interesse ultimo" dell'uomo (ultimate concern), manifestando un'estrema libertà nel passaggio dall'utilizzo di nozioni metafisiche a categorie psicologiche (e proprio in questo passaggio si situa il centro del pensiero di Tillich) [...]. "Dio" è il nome attribuito all'“interesse ultimo" dell'uomo; ma una tale riduzione psicologica è esattamente ciò che veniva chiamato ateismo nel XIX secolo da Feuerbach, il quale riteneva che il linguaggio religioso non fosse privo di un referente reale, sebbene esso avesse carattere puramente umano [per non dire "troppo umano", secondo il celebre motto di Nietzsche $]^{18}$.
\end{abstract}

Un immotivato, quanto affrettato, rigetto della tradizionale metafisica-teologia razionale viene infine imputato - da Maclntyre - a Hans Küng, il quale, nel tentativo di fornire argomenti a favore dell'esistenza di Dio, capaci di resistere alla corrosione della

\footnotetext{
${ }^{15}$ A. MACINTYRE, The Debate about God, in A. MACINTYRE - P. RICOEUR, The Religious Significance of Atheism, Columbia U.P., New York 1969, pp. 1-55 (spec. p. 25, corsivo aggiunto).

${ }^{16}$ G. VATTIMO, Dopo la cristianità, Garzanti, Milano 2002, p. 71.

${ }^{17}$ Cfr. A. MACINTYRE, God and the Theologians, cit., p. 23.

${ }^{18}$ A. MACINTYRE, The Debate about God, cit., pp. 27-8.
} 
moderna secolarizzazione, afferma sic et simpliciter che la fiducia nella "realtà incerta" e nella "razionalità umana" è del tutto ingiustificata se si esclude l'esistenza di Dio:

[Così facendo, tuttavia,] Küng aggiunge soltanto un'ulteriore tessera alla nostra già ampia collezione di credenze infondate, poiché non avanza mai un criterio di giustificazione adeguato per la peculiare credenza nell'esistenza di Dio [...]. Küng, quindi, non fornisce alcuna buona ragione per credere nell'esistenza di Dio [...] né discute mai quale carattere debba essere attribuito alla giustificazione della credenza religiosa ${ }^{19}$

MacIntyre conclude che maggiore credito meritano coloro che hanno riscoperto e approfondito, nell'ambito della scuola analitica, i tradizionali argomenti della teologia razionale, completamente ignorati da Küng, vale a dire Peter Geach, Antony Kenny, Terence Penelhum, Alvin Plantinga, Richard Swinburne, et al., ai quali, in qualche misura, egli tributa a posteriori l'abbandono di quel "fideismo barthiano", che aveva caratterizzato gli esordi della propria riflessione sull'esperienza religiosa, come si evince altresì dal tenore di una celebre intervista concessa a qualche anno di distanza:

\begin{abstract}
Per un certo periodo, [... trattai la pratica religiosa] come una forma di vita sui generis, mescolando una peculiare interpretazione del concetto di "forma di vita" avanzato da Wittgenstein e la teologia di Barth. Ma presto mi resi conto che le rivendicazioni implicite nell'uso del linguaggio e della pratica religiosi sono inseparabili da una serie di sottintesi metafisici, scientifici e morali [...]. Quando giunsi a rifiutare la strana mescolanza filosofica di un malinteso Wittgenstein e di un Barth troppo preso alla lettera, [...] parti di tomismo sopravvissero nel mio pensiero insieme ad alcune riflessioni più ponderate su Wittgenstein ${ }^{20}$.
\end{abstract}

\title{
La "teologia post-secolare" di John Milbank
}

L'intento di Maclntyre di salvaguardare - sotto l'egida di un inedito neotomismo, che fa proprie talune acquisizioni della moderna filosofia del linguaggio, dell'ermeneutica, ecc. - i loci theologici tradizionali, relativi alla conoscenza naturale di Dio, è stato aspramente criticato da Milbank, iniziatore del movimento teologico della

\footnotetext{
${ }^{19}$ A. MAcInTYRe, Dr. Küng's Fiasco. Review of Hans Küng, Does God Exist?, in «London Review of Books», 3 (1981), pp. 7-8 (spec. p. 8).

${ }^{20}$ G. BORRADORI, Conversazioni americane, Laterza, Roma-Bari 1991, p. 174.
} 
radical orthodoxy ${ }^{21}$ : quest'ultimo, infatti, nonostante condivida il deciso rifiuto macintyriano opposto a qualunque fattispecie di teologia della secolarizzazione, dubita che il recupero degli standards di razionalità, propri dell'etica delle virtù e della tradizione aristotelico-tomista, possa avere la meglio, nel confronto dialettico con l'ideologia secolare, liberale e postmoderna. Ciò che - a suo dire - è necessario per superare la "ragione secolare" è qualcosa che sia esteticamente più accattivante, piuttosto che una visione più razionale del mondo e della natura umana:

MacIntyre vuole argomentare contro le tendenze stoico-liberali-nichilistiche, che costituiscono in sé la "ragione secolare". Poiché, tuttavia, quest'ultima è soltanto un mito, non può essere confutata, bensì meramente sostituita, nella misura in cui è possibile persuadere i propri interlocutori - tramite l'appagamento del loro "gusto letterario" [literary taste] - che il cristianesimo offre una narrazione migliore ${ }^{22}$.

I movimenti che avvengono tra una tradizione e l'altra - ad esempio da una concezione aristotelica della natura ad una newtoniana - devono infatti essere interpretati sostanzialmente come "vittorie retoriche":

Il primato accordato all'apprendistato e alla tradizione, all'interno dello schema neoplatonico e poi cristiano di comprensione del logos, porta a relazionare l'esercizio delle virtù con un abito mentale di tipo retorico piuttosto che dialettico, con un logos che privilegia l'opinione (doxa), la testimonianza (marturia) e la persuasione (pistis). Questo legame della retorica con la causa del cristianesimo - conclude Milbank - è misconosciuto da Maclntyre, che associa la retorica esclusivamente alla perdita della trascendenza, all'oblio delle virtù e all'emergente individualismo ${ }^{23}$.

${ }^{21}$ Cfr. sulla genesi del movimento e sulle sue varie affiliazioni, cfr. J. MILBANK - C. PICKSTOCK - G. WARD (eds.) Radical Orthodoxy, Routledge, London-New York 1999; L.P. HEMMING (ed.), Radical Orthodoxy. A Catholic Enquiry, Ashgate, Aldershot 2000; G. HyMAN, The Predicament of Postmodern Theology: Radical Orthodoxy or Nihilist Textualism?, Westminster John Knox, Louisville 2001; J.K.A. SMITH, Introducing Radical Orthodoxy: Mapping a Post-Secular Theology, Baker Academic, Grand Rapids 2004; W.J. HANKEY - D. HEDLEY (eds.), Deconstructing Radical Orthodoxy: Postmodern Theology, Rhetoric, and Truth, Ashgate, Aldershot 2005; M. SALVIOLI, Verso un tomismo post-secolare. Milbank interprete di Tommaso d'Aquino, Angelicum U.P., Roma 2014, ecc.

22 J. MilBANK, Theology and Social Theory: Beyond Secular Reason (1990), Blackwell, Malden $2006^{2}$, p. 331.

${ }^{23}$ Ibid., p. 329. Milbank, pertanto, aggiunge: «In contrast to most critique of Maclntyre, I do not find him sufficiently relativistic or historicist [...]; I do not hitch the cause of virtue to the cause of Socratic dialectics in the way that Maclntyre does (much more in Whose Justice? Which Rationality? than in earlier After Virtue). Indeed, part of the project of this chapter could be described as "the detachment of virtue from dialectics"» (p. 328). Riguardo alla distinzione tra un'impostazione epistemologica "dialettico- 
La ragione secolare, del resto, forgiata dagli epigoni del nichilismo nietzschiano, si rivela un mito, tanto più insoddisfacente, quanto più implica la violenza e la prevaricazione, ovvero un'incessante lotta per l'autoaffermazione, che si perpetua inesorabilmente nella vittoria del più forte. Ora, pur non essendo possibile ricorrere a criteri di giustificazione razionale sub specie aeternitatis - che siano indipendenti dalla loro vis rhetorica e dall'esser costituiti da una tradizione di appartenenza -, la teologia cristiana, basata sulla concezione della creazione divina, è comunque capace di attingere ad un "realismo metanarrativo", che abbraccia intenzionalmente ogni aspetto della vita umana e libera dal nichilismo e dalla violenza: «La logica cristiana — ad avviso di Milbank - non è decostruibile dalla moderna razionalità secolare; al contrario, il cristianesimo mostra che non è necessario supporre, come fanno i nietzschiani, che la differenziazione non totalizzante e l'indeterminatezza dei significati implichino inevitabilmente l'arbitrarietà e la violenza» ${ }^{24}$.

È la stessa concezione di Dio - che Milbank ritiene di dover mutuare dalla patristica, in particolare da Dionigi l'Areopagita, Massimo il Confessore, Agostino, et al. - che autorizza il cristianesimo non solo a tollerare, bensì a comporre in unità le differenze: Dionigi, ad esempio, definisce Dio quale "Essere sovrabbondante" da cui scaturisce la molteplicità degli esseri viventi, ma anche l'armonia all'interno del creato.

L'“unità di Dio" è quindi sinonimo di una "pace trascendentale", che riequilibra e armonizza le differenze e le processioni divine: «La teologia cristiana, dunque, si contrappone al nichilismo nietzschiano, che contempla la violenza fondativa, poiché istituisce una grammatica della partecipazione e dell'analogia, una semiotica della pace, una metanarrativa che non richiede il postulato della violenza primordiale ${ }^{25}$.

obiettivistica" e una "retorico-relativistica", che si confrontano nell'ambito dell'odierna teologia cristiana, cfr. T. JACOBS, The Problem of a Postmodern (Theological) Epistemology, or the Temptation of a Narrative Ontotheology, in M. LAMBERIGTS - L. BoeVE - T. MeRRIGAN, Theology and the Quest for Truth, Leuven U.P., Leuven 2006, pp. 61-76.

${ }^{24}$ Ibid., p. 6.

${ }^{25}$ A. McGrath, A Scientific Theology: Reality, Vol. 2, T\&T Clark, London 2006², pp. 105 ss. 
Naturalmente, sono numerose le critiche rivolte all'indirizzo di Milbank, a partire dall'obiezione che la sua teologia post-secolare difficilmente può essere identificata con il cristianesimo tout court ${ }^{26}$ : «La narrazione, che costituisce il fulcro del cristianesimo, potrebbe essere interpretata nel modo che Milbank ritiene più persuasivo, ma essa non richiede necessariamente di essere interpretata così» ${ }^{27}$. E ciò anche in considerazione del fatto che la cristologia di Milbank appare a molti colleghi evanescente, poiché, da un lato, egli afferma che Gesù è essenzialmente una realtà linguistica e poetica ${ }^{28}$, e, dall'altro, simili «aperture ad alcune concezioni postmoderne della filosofia del linguaggio sembrano spingerlo in direzioni che contrastano, de facto, con le concrete narrazioni e pratiche cristiane ${ }^{29}$.

La categoria del realismo metanarrativo - a cui allude Milbank - rimane, quindi, in ultima istanza, assai controversa: egli - nell'introduzione a Theology and Social Theory (1990), manifesto programmatico della radical orthodoxy - pur rifiutando il "realismo filosofico" di Maclntyre, a vantaggio di un "idealismo linguistico" e di una "variante del pragmatismo", dichiara infine di adottare "una posizione critica della modernità, che è sì storicista e pragmatista, ma, allo stesso tempo, fautrice di un "realismo teologico", in linea con il pensiero di Maurice Blondel»"

La confusione emerge laddove Milbank sostiene di abbandonare il realismo filosofico, a beneficio di un presupposto realismo teologico, senza però spiegare adeguatamente l'ubi consistam di quest'ultimo passaggio. Ciò induce a ritenere che il suo discorso non sia conclusivo: "Fintanto che il realismo teologico di Milbank non sarà meglio esplicitato, l'“integrità teologica” della sua opera apparirà in pericolo»”, e il

${ }^{26}$ Cfr. G. RICHARDSON, Integrity and Realism: Assessing John Milbank's Theology, in "New Blackfriars», 84 (2003), pp. 268-80, che contiene un'accurata rassegna critica rivolta all'opera di Milbank.

${ }^{27}$ G. HYMAN, The Predicament of Postmodern Theology, cit., p. 88 (corsivo aggiunto).

${ }^{28}$ Cfr. J. MILBANK, The Word Made Strange: Theology, Language, Culture, Blackwell, Oxford 1997, p. 3.

29 F.C. BAUERSCHMIDT, The Word Made Speculative? John Milbank's Christological Poetics, in "Modern Theology», 15 (1999), pp. 417-32 (spec. p. 429).

30 J. MILBANK, Theology and Social Theory, cit., p. 6.

${ }^{31}$ G. RICHARDSON, Integrity and Realism, cit., p. 279. 
realismo filosofico di Maclntyre risulterà, comunque, maggiormente in sintonia con una visione cristiana del mondo e dell'esperienza umana.

\section{"Narratività": nuova panacea dei problemi teologici e morali?}

La reazione alla "teologia liberale" - di cui la "teologia della secolarizzazione" può essere considerata l'extrema ratio - ha assunto molteplici forme: "teologia narrativa", "teologia della prassi", "radical orthodoxy", ecc., le quali, però, convengono essenzialmente nel valorizzare un "ritorno alla Scrittura", al fine di superare l'approccio rigidamente "proposizionale-cognitivistico" - imputato tanto alla dogmatica neoscolastica, quanto all'esegesi razionalista - a favore di un "pragmatismo scritturale" ${ }^{32}$, radicato nella prassi di vita di una comunità interpretativa e di una tradizione storica, qual è quella ebraico-cristiana, seppur nella legittima pluralità delle sue espressioni.

Ebbene, i principali caratteri, che contraddistinguono la "teologia narrativa postliberale" ${ }^{\prime 33}$, sono così riassumibili: 1) una concezione estesa di razionalità, che include

32 Il termine è stato coniato da C.C. PECKNOLD (Transforming Postliberal Theology: George Lindbeck, Pragmatism and Scripture, T\&T Clark, London 2005), al fine di definire lo stile teologico di Lindbeck, esponente di punta della teologia postliberale.

${ }^{33}$ Cfr. J. FODOR, Postliberal Theology, in D. FORD - R. MUERS (eds.), The Modern Theologians, cit., pp. 229-48: «Postliberal theology names an internally highly differentiated movement in contemporary Englishspeaking theology (primarily in North America and Britain) [...]. George Lindbeck and Hans Frei are the two seminal figures of this distinctive kind of theological engagement. Other exponents - and this is a representative not an exhaustive list - include Stanley Hauerwas, Ronald Thiemann, James Buckley, Joseph Di Noia, Garrett Green, George Hunsinger, William Werpehowski, Bruce Marshall, William Placher, Kathryn Greene-McCreight, Serene Jones, Joseph Mangina, Eugene Rogers, and Kathryn Tanner. In its more recent developments and permutations the list may be extended to include, on the one hand, figures like John Milbank, Catherine Pickstock, and Graham Ward and, on the other, Peter Ochs, David Ford, and Daniel Hardy. To be sure, not all of the above would feel entirely comfortable accepting the appellation "postliberal" as a self-description, nor would they necessarily see themselves being classified together, let alone advancing a common cause [...]. The intellectual influences shaping and informing postliberal theology are as diverse and varied as are its practitioners. Major impetuses include but are not limited to: the theology of Karl Barth; new appropriations of Martin Luther and Thomas Aquinas (principally inspired by the nouvelle théologie: a renewal within the Catholic Church to return critically to the sources of the faith in scripture and premodern tradition in light of contemporary needs); developments in the philosophy of science, especially the work of Thomas Kuhn and Michael Polanyi; new directions in philosophy of language indebted to Ludwig Wittgenstein and Gilbert Ryle; an appreciation of recent sociological analyses and insights, principally those of Peter Berger and Robert Bellah; advances in the field of anthropology, specifically Clifford Geertz; recent work in narrative and narrative analysis from literary (Eric Auerbach, 
anche la dimensione contestuale e storicamente determinata della ragione umana; 2 ) un riconoscimento del ruolo insostituibile che le narrazioni e i testi fondativi svolgono nel forgiare la razionalità e la cultura umane; 3) una rivalutazione - in antitesi con l'individualismo liberale - delle relazioni genetiche, che ogni essere umano intrattiene con la comunità di appartenenza, all'interno del processo di costruzione dell'identità personale; 4) una valorizzazione della "riserva escatologica" del cristianesimo.

Figure seminali di questo movimento teologico, diffusosi prevalentemente all'interno di università anglosassoni, sono Hans Frei, autore dell'influente The Eclipse of Biblical Narrative (1974), recensito da Maclntyre quale saggio capace di rivoluzionare i canoni ermeneutici biblici ${ }^{34}$, e George Lindbeck, autore di The Nature of Doctrine: Religion and Theology in a Postliberal Age (1984), da cui deriva la convenzionale denominazione del movimento stesso.

Il debito contratto nei confronti di Barth, dalla teologia narrativa postliberale, è illustrato nella monografia di David Ford, Barth and God's Story (1985), da cui si evince come, a partire dal Commento alla Lettera ai Romani, la crocifissione e la resurrezione di Cristo siano utilizzate dal teologo svizzero per differenziare il cristianesimo da qualsiasi altra "religione umana": il Dio dei racconti della crocifissione e resurrezione «non è più Qualcuno che i cristiani possono assumere di avere in comune con altri».

La conseguenza capitale che ne discende è che "Dio stesso può essere ora descritto soltanto attraverso tali racconti» (Deus ipse narrativus) ${ }^{35}$ ! Pertanto, la principale preoccupazione della teologia narrativa postliberale diviene la fedele autodescrizione del cristianesimo e della storia della salvezza, piuttosto che la loro

Frank Kermode), biblical (Michael Fishbane, Moshe Greenberg), and philosophical (Alasdair Maclntyre) perspectives. Postliberal theology is occasionally referred to as intratextual or narrative theology, given its crucial emphasis on biblical narrative. It is also sometimes called the Yale school or the new Yale school of theology, historically signaling one of its important wellsprings: Yale University Divinity School» (spec. pp. 229 ss.).

34 Cfr. A. MAcInTYRE, Review of Hans W. Frei, The Eclipse of Biblical Narrative, in "The Yale Review», 65 (1976), pp. 251-55.

35 D. FORD, Barth and God's Story: Biblical Narrative and the Theological Method of Karl Barth in the Church Dogmatics, Peter Lang, Frankfurt-Bern-New York 1985, p. 21. 
correlazione con le domande universali che ogni essere umano si pone riguardo al significato ultimo dell'esistenza.

Il linguaggio è dunque primariamente compreso in funzione dossologica, piuttosto che in ragione della propria corrispondenza con la realtà: la narrazione biblica definisce una sorta di dominio linguistico di riferimento, capace di generare una "koinè culturale", una visione del mondo che si traduce in prassi comune, propriamente liturgica ${ }^{36}$. II corpus dottrinale è, infine, una descrizione di "secondo livello", costruita sulla base di quell'universo scritturale e semiotico, esperito all'interno del vissuto storico delle comunità ebraico-cristiane ${ }^{37}$.

Vi sono ciononostante quattro aree - che sono sin qui rimaste sullo sfondo nelle quali il dibattito è tutt'oggi aperto tra i fautori della teologia narrativa postliberale, ovvero: 1) la possibilità di un accesso adeguato alla realtà, vale a dire in una modalità non relativistica; 2) i criteri che possano permettere di descrivere in maniera epistemologicamente appropriata la relazione che intercorre tra i testi fondativi e la comunità interpretante; 3) la coerenza delle narrazioni presenti nella Scrittura e nella tradizione, ossia la determinazione del tasso di pluralismo ermeneutico che è possibile considerare fisiologico, in merito all'interpretazione delle suddette narrazioni; 4) il rischio incombente di settarismo e quindi la ricerca di un'autentica definizione, fedele al testo biblico, del rapporto tra comunità di fede e mondo secolarizzato. In verità, si può affermare che, lungi dal costituire una nuova panacea dei problemi filosofici e teologici, I'intento della teologia narrativa postliberale è eminentemente correttivo, anziché fondativo.

\footnotetext{
${ }^{36}$ Cfr. C. PICKSTOCK, After Writing: The Liturgical Consummation of Philosophy, Blackwell, Oxford 1997 e i commenti di F. KeRR, A Catholic Response to the Programme of Radical Orthodoxy, in L.P. HeMmING (ed.), Radical Orthodoxy, cit., pp. 46-59.

37 Cfr. J. FODOR, Postliberal Theology, cit., p. 231: "The theologian investigates the first-order use of scripture in the faith community (in worship, prayer, preaching, catechesis, piety, and life) in order to generate "second-order concepts and theories which make maximum sense of these actual practices" [G. LINDBECK, Atonement and Hermeneutics of Intratextual Social Embodiment, in T.R. PHILLIPS - D.L. OKHOLM, eds., The Nature of Confession: Evangelicals and Postliberals in Conversation, InterVarsity, Downers Grove 1996, pp. 221-40, spec. p. 222]».
} 
[Del resto,] mentre riescono normalmente ad evitare un'adozione irriflessa della narrazione in sé (l'idea che la forma narrativa abbia in sé e per sé una speciale significanza cognitiva), i teologi postliberali non hanno ancora precisato con sufficiente chiarezza il ruolo svolto dal discorso sistematico all'interno della teologia, di modo che non leda la loro insistenza sull'irrinunciabilità della forma narrativa [nell'ambito della rivelazione cristiana e della cultura umana]. È vero, infatti, che il testo narrativo è capace di presentare oggetti che non potrebbero essere presentati in altra forma, ma questo non è un argomento conclusivo a favore dell'autosufficienza della narrazione [...]. Poiché la fede cristiana produce anche affermazioni di rilevanza universale, la teologia deve essere in grado di padroneggiare "gli schemi astratti del discorso sistematico", così come "le insostituibili specificità del discorso narrativo", senza considerare il primo discorso come mero epifenomeno del secondo [...]. II rigetto del fondazionalismo non deve necessariamente sfociare in un abbandono del discorso sistematico, né nell'evasione dalle domande metafisiche implicite in tale discorso, [né, tanto meno, nella dissoluzione della filosofia all'interno della teologia]. Al contrario, poiché la trattazione di questioni teoretiche ed epistemologiche è elemento costitutivo della teologia, concedere, che un approccio culturale-linguistico qual è proprio della teologia postliberale - meramente autorizzi, ma non richieda di per sé alcun realismo cognitivo, potrebbe risultare insufficiente: la teologia postliberale deve essere maggiormente persuasiva, offrendo ragioni convincenti riguardo alle conseguenze che comporta l'opzione, o meno, a favore di un solido realismo cognitivo ${ }^{38}$.

"Realismo cognitivo" che costituisce - ad avviso di Maclntyre - il maggior cespite della tradizione aristotelico-tomista, con il quale ogni altra tradizione di pensiero filosofico e teologico è chiamata incessantemente a misurarsi ${ }^{39}$.

${ }^{38}$ Ibid., p. 238 (corsivo aggiunto). Il dibattito sui guadagni e sui limiti della teologia narrativa è, come si è detto, tuttora in corso: per una ricostruzione storica dettagliata rinvio a G.W. STROUP, The Promise of Narrative Theology, John Knox, Atlanta 1981; G.L. CомSTOcK, Two Types of Narrative Theology, in «Journal of the American Academy of Religion», 55 (1987), pp. 687-717; S. HAUERWAS - L. GREGORY JONES, Why Narrative? Readings in Narrative Theology, Eerdmans, Grand Rapids 1989; G. LougHLIN, Telling God's Story: Bible, Church and Narrative Theology, Cambridge U.P., Cambridge 1995; V. ANDERSON, The Narrative Turn in Christian Ethics: A Critical Appraisal, in "American Journal of Theology and Philosophy», 19 (1998), pp. 293-312; K.E. YANDELl (ed.), Faith and Narrative, Oxford U.P., Oxford 2001; P.J. DeHART, The Trial of the Witnesses: The Rise and Decline of Postliberal Theology, Blackwell, Malden 2006; A. LUCIE-SMITH, Narrative Theology and Moral Theology: The Infinite Horizon, Ashgate, Aldershot 2007; F.A. MuRPHY, God is Not a Story, cit., ecc.

${ }^{39}$ Cfr. A. MACINTYRE, Philosophy Recalled to Its Tasks: A Thomistic Reading of Fides et Ratio, in ID., The Tasks of Philosophy: Selected Essays, Vol. 1, Cambridge U.P., Cambridge 2006, pp. 179-96: "It follows that any type of philosophy that is to be able to function as philosophy must function, if it is to achieve its own ends, may be nonThomistic or even antiThomistic in many respects, [...] but that they will have to find some place for those truths that were classically articulated as the doctrine of Thomistic realism » (spec. p. 192, corsivo aggiunto). Del resto, lo stesso A. MACINTYRE (Three Rival Versions of Moral Enquiry, University of Notre Dame Press, Notre Dame 1990, p. 67), rileva come il realismo cognitivo sia intimamente teistico: «[Realism] has at its core the view that the world is what it is independently of human thinking and judging and 


\title{
Racconto fondatore e radicamento ontologico
}

Un teologo di formazione neotomista, contemporaneo di Maclntyre, Ghislain Lafont, ha prospettato in Dieu, le temps et l'être (1986) l'ineludibilità di un'“eteronomia fondatrice", basata su due principi distinti, ma intimamente correlati: la narratività e l'analogia; ma potremmo egualmente dire: la storia e l'essere, l'ermeneutica e la metafisica, ecc.

Parlare di Dio, in termini narrativi, come di un evento, abitudine oggi molto diffusa, può esser fatto - sostiene Lafont - a condizione di essere pronti ad analizzare criticamente l'uso teologico di questo vocabolo, per non cadere vittime di un'apparente evidenza del concetto (che cos'è in realtà un evento?) e di una certa ingenuità nell'attribuzione di questo nuovo "nome divino". Provocati da una serie di eventi, di cui la fede organizza la tradizione alla luce del Mistero pasquale di Gesù e in cui essa riconosce la rivelazione progressiva di Dio agli uomini, si è giunti, infatti, a riconoscere in Dio stesso delle "relazioni-processioni interne", definite talvolta esse stesse eventi:

\begin{abstract}
Ma quali sono le sfumature che deve assumere il termine quando lo applichiamo successivamente e contemporaneamente: a degli avvenimenti nel tempo, come quello di Davide o la morte di Gesù di Nazareth; ad altri che sembrano insieme nel tempo e al di fuori, come il passaggio del Mar Rosso e la risurrezione di Gesù; all'azione propriamente detta di "Dio" che interviene (come e in che modo?) per fare accadere questi eventi; alla vita stessa di Dio, infine, se è possibile parlare in sé e per sé del soggetto trascendente degli atti della salvezza? A leggere queste domande, tanto inevitabili quanto semplici, sembra che il termine evento sia un termine analogico. La serie di eventi che noi chiamiamo "storia della salvezza" è continua nel suo orientamento, ma discontinua nella densità di ogni suo momento, diversa nelle modalità di azione di Dio, le quali danno luogo a dei nomi divini che non sono sinonimi [...]. Se si tralascia, quando si parla di evento, di riconoscere l'analogia del termine, non si fanno delle confusioni che mettono in pericolo il processo narrativo stesso? Tener conto dell'analogia è necessario alla verità della narrazione $e^{40}$.
\end{abstract}

desiring and willing. There is a single true view of the world and of its ordering, and for human judgments to be true and for human desiring and willing to be aimed at what is genuinely good they must be in conformity with that divinely created order».

${ }^{40}$ G. LAFONT, Dieu, le temps et l'être, Éditions du Cerf, Paris 1986 (trad. it., Dio, il tempo e l'essere, Piemme, Casale Monferrato 1992, pp. 179-80). 
Non si tratta, quindi, di un esercizio teoretico-metafisico facoltativo, all'interno di un percorso teologico che rimane, ciononostante, eminentemente narrativo; al contrario, lo stesso sviluppo narrativo della storia della salvezza perde la sua coerenza interna (e peggio, forse, la sua stessa intelligibilità), se una parola appropriata non viene pronunciata per suggerire il livello ontologico di Dio in se stesso, quale soggetto trascendente degli eventi di salvezza.

Lafont è ben consapevole che voler reintrodurre oggi l'"argomento dell'essere" in teologia è impresa delicata, sospetta, quasi disperata, eppure quanto mai necessaria: mentre, infatti, in ambito analitico, la teologia razionale e il discorso sull'"essere di Dio", conoscono una nuova stagione di rinascita, in ambito continentale, prevale ancora - con le dovute eccezioni: Lafont docet - un indirizzo heideggeriano che ambisce a concepire, alla maniera di Jean-Luc Marion, Dieu sans l'être (1982), ovvero senza le idolatrie concettuali della metafisica e dell'onto-teologia, che vogliono imprigionare Dio all'interno delle categorie logico-razionali umane ${ }^{41}$.

\footnotetext{
${ }^{41}$ Per un'illustrazione sintetica del pensiero teologico di Marion, che fa perno su una "fenomenologia del dono", cfr. B. RoBINETTE, A Gift to Theology? Jean-Luc Marion's "Saturated Phenomenon" in Christological Perspective, in "The Heythrop Journal», 48 (2007), pp. 86-108: "To think God "without Being" is not to cease thinking or philosophizing, but to allow thought itself to be penetrated and disturbed by the free, revelatory gift of God, as it gives itself. "God can give himself to be thought without idolatry only starting from himself alone: to give himself to be thought as love, hence as gift; to give himself to be thought as a thought of the gift" [J.-L. MARION, God without Being, 1982, The University of Chicago Press, Chicago 1991, p. 49]. As Marion thinks God as gift, as unsuspected agape overflowing all thought, he expressly appeals to the likes of Pseudo-Dionysius and Bonaventure who prioritize the self-diffusive Good over Being. God gives Being to beings. Being itself is determined by the anterior freedom of the one who gives. This anteriority of freedom reveals the infinite "distance" between God and beings, a distance not traversable by thought. And yet, through the distance, revelation finds us. It finds us in the startling self-bestowal of God in Christ, the image (or Icon) of the invisible God. The directional difference is decisive: in the Idol, we aim at the divine, but end up gazing at ourselves; in the Icon of Christ, our own gaze is reversed by the divine gaze who envisages us. As we shall see, this theology of Christ as Icon is the "saturated phenomenon" par excellence, where God's self-giving exceeds vision with a light so overwhelming that it becomes a darkness: or, what Pseudo-Dionysius describes as "dazzling darkness"» (spec. pp. 88-9). J.-L. MARION (Saint Thomas d'Aquin et l'onto-théo-logie, in "Revue Thomiste», 95, 1995, pp. 31-66) ha attenuato in parte il proprio giudizio negativo sulla teologia razionale dell'Aquinate: cfr., al riguardo, B.J. SHANLEY (St. Thomas, OntoTheology, and Marion, in «The Thomist», 60, 1996, pp. 617-25), il quale propone altresi un inedito paragone: «lt nevertheless seems possible that Marion may turn out to be something like Alasdair Maclntyre: an established philosopher trained outside the Thomistic tradition, initially critical of Thomas, who eventually finds his way to embracing Thomas in provocative and innovative ways» (spec. p. 625).
} 
Tuttavia, un Dio concepito come "totalmente altro", un "Dio senza essere", può rivelarsi radicalmente alieno dall'esistenza e dalla storia dell'umanità, "a tal punto distante, da sembrare defunto ${ }^{42}$; rendendo così estremamente difficile (per non dire impossibile), discernere e separare I'“essere di Dio" (Godself) da quello di un "sublime mostro", la rivelazione divina dal mito, la vera fede dalla pazzia, ecc. ${ }^{43}$ Mentre simili "discrasie teologiche" costituiscono l'esito paradossale di quell'"oblio dell'essere" che Heidegger intendeva superare, una "teologia dell'eteronomia" che voglia coniugare correttamente narratività, prassi cristiana e radicamento ontologico - ciò di cui non sembra capace la teologia postmoderna che si ispira al filosofo tedesco - potrà senz'altro giovarsi del contributo teoretico offerto da Maclntyre e dal tomismo analitico.

\section{Maclntyre: "tomista analitico" o "tomista agostiniano postmoderno"?}

È doveroso, avviandoci alla conclusione, riassumere concisamente quale sia stata l'eco della teoria morale (e della coeva teologia filosofica) di Maclntyre nel vasto panorama della teologia cristiana contemporanea. Ebbene, i riferimenti all'etica delle virtù sono ampiamente diffusi (e, spesso, entusiastici) nell'ambito della teologia morale:

II metodo particolare dell'etica delle virtù costituisce, infatti, un contesto privilegiato per i teologi morali, al fine di collaborare insieme all'indagine riguardante un ampio numero di questioni disciplinari [...]. Tale teoria etica è tanto conveniente per la teologia morale cattolica odierna quanto lo fu l'Etica Nicomachea di Aristotele per la Summa Theologiae di s. Tommaso ${ }^{44}$ !

\footnotetext{
${ }^{42}$ Cfr. R. KeARNEY, The God Who May Be: A Hermeneutics of Religion, Indiana U.P., Bloomington 2001, p. 79.

${ }^{43}$ Cfr. R. KEARNEY, Strangers, Gods, and Monsters: Ideas of Otherness, Routledge, London-New York 2003.

44 J.F. KeENAN, Theological Trends. Christian Ethics: The Last Ten Years, in "The Way», 32 (1992), pp. 215224 (spec. p. 221). Numerosa letteratura può suffragare la tesi di Keenan: cfr. F. KERR, Moral Theology after MacIntyre: Modern Ethics, Tragedy, and Thomism, in "Studies in Christian Ethics», 8 (1995), pp. 33-44; J.J. KotVA, The Christian Case for Virtue Ethics, Georgetown U.P., Washington D.C. 1997; N. MURPHY - B.J. KAlleNBerg - M. ThIESSEN NATION (eds.), Virtues \& Practices in the Christian Tradition: Christian Ethics after MacIntyre, Trinity Press International, Harrisburg 1997; J.R. WILSON, Living Faithfully in a Fragmented World: Lessons for the Church from Maclntyre's After Virtue, Trinity Press International, Harrisburg 1997; D.J. HARRINGTON - J.F. KEENAN, Jesus and Virtue Ethics: Building Bridges between New Testament Studies and Moral Theology, Rowman \& Littlefield, Lanham 2002; J. McEvor, Parallel Projects: Alasdair Maclntyre's Virtue Ethics, Thirteenth-Century Pastoral Theology (Leonard Boyle, O.P.), and Thomistic Moral Theology (Servais Pinckaers, O.P.), in F. O'RouRKe (ed.), What Happened In and To Moral Philosophy in the Twentieth Century? Philosophical Essays in Honor of Alasdair Maclntyre, University of Notre Dame Press, Notre Dame 2013, pp. 244-70, ecc.
} 
Ma anche nella "teologia fondamentale" e nella "teologia dogmatica" i riflessi del pensiero macintyriano si sono avvertiti, sebbene in misura certamente meno eclatante: basti ricordare l'attenzione riservata a Maclntyre da parte di John Milbank, portavoce della "radical orthodoxy", e di Tracey Rowland, teologa che definisce se stessa (e Maclntyre) quale "tomista agostiniana postmoderna" (postmodern Augustinian Thomist):

\begin{abstract}
Sebbene Maclntyre sia stato variamente ritratto con i seguenti appellativi: "comunitarista", "virtue-ethicist", "aristotelico rivoluzionario", "tomista romantico" e "tomista postmoderno", la più soddisfacente categorizzazione della sua posizione filosofica è quella di "tomista agostiniano postmoderno" un esempio di ciò che Graziano e i canonisti medievali definivano come concordantia discordantium canonum - una sintetica riconciliazione di principi apparentemente rivali [...]. Qui, l'aggettivo "agostiniano" aiuta a conferire l'idea che sia fondamentale - per poter efficacemente operare una sintesi [con il pensiero (post-)moderno] — l'interesse rivolto ad argomenti tipicamente agostiniani: il rapporto tra ordine sacrale e ordine secolare; il ruolo della memoria nell'edificazione dell'anima e l'importanza della tradizione narrativa per la crescita intellettuale e spirituale ${ }^{45}$.
\end{abstract}

La Rowland riconosce principalmente al filosofo scozzese il merito dello "sviluppo della tradizione tomista" nella direzione auspicata dalla scuola della Nouvelle Théologie, in linea con il pensiero di Henri de Lubac, Hans Urs von Balthasar, et al., e il loro rinnovato interesse per lo studio della patristica (e di Agostino, in particolare).

L'“approccio balthasariano” - condiviso, a giudizio dell'Autrice, anche da Maclntyre - si propone, infatti, di ricostruire l'“unità sinfonica” del vero, attraverso la cucitura di un ricco ordito, intessuto di un insieme composito di biografia, poesia, storia, analogie trinitarie, analisi logico-linguistica, verità rivelate, ecc. ${ }^{46}$ Nonostante le severe critiche mosse da Milbank all'indirizzo di Maclntyre, la Rowland ritiene, altresì, che tale approccio sia congruente con il metodo esperito dalla radical orthodoxy per "unire

\footnotetext{
${ }^{45}$ T. RowLAND, Culture and the Thomist Tradition after Vatican II, cit., pp. 5-6. Contra, cfr. J.A. KOMONCHAK, "A Postmodern Augustinian Thomism?", in L BOEVE - M LAMBerigTS - M. WISSE (eds.), Augustine and Postmodern Thought: A New Alliance against Modernity?, Peeters, Leuven 2009, pp. 123-46.

${ }^{46}$ Cfr. H.U. VON BALTHASAR, Die Wahrheit ist symphonisch. Aspekte des christlichen Pluralismus, Johannes, Einsiedeln 1972 (trad. it., La verità è sinfonica. Aspetti del pluralismo cristiano, Jaca Book, Milano 1991).
} 
l'esegesi e la riflessione culturale-filosofica in un complesso, ma coerentemente eseguito, collage ${ }^{47}$.

L'Autrice sostiene, in ultima istanza, la preminenza euristica dell'impostazione teologica "continentale-balthasariana" (Continental Balthasarian), rispetto al modello rivale del "tomismo analitico" (Anglo-American Analytical Thomist), poiché, "grazie alla disponibilità a comprendere la genesi storica delle questioni filosofico-teologiche e alla priorità accordata all'“analisi della logica delle tradizioni”, piuttosto che all'“analisi linguistica dei termini" da queste impiegati, è meglio posizionata per affrontare la prova della "crisi [postmoderna]" ${ }^{48}$.

Ora, al di là di qualunque ruolo effettivo che l'Aquinate possa rivestire nell'“ordito sinfonico" di von Balthasar - secondo Fergus Kerr, egli in alcune parti della sua opera dà piuttosto l'impressione di considerare Tommaso sostanzialmente come una "figura transitoria" ${ }^{49}$, il che lo porrebbe inevitabilmente in antitesi con Maclntyre sono persuaso che la lettura proposta dalla Rowland, riguardo al tomismo analitico, non

\footnotetext{
${ }^{47}$ J. MILBANK - C. PICKSTOCK - G. WARD (eds.), Radical Orthodoxy, cit., p. 2; non è un caso quindi che il volume della Rowland sia stato pubblicato nella collana "Radical Orthodoxy Series", edita dagli stessi curatori qui menzionati.

${ }^{48}$ T. RoWLAND, Culture and the Thomist Tradition after Vatican II, cit., p. 163 (corsivo aggiunto). L'Autrice cita per suffragare la rivalità (o meglio l'incompatibilità) tra le due prospettive, un breve e conciso scambio dialettico intercorso tra J. HALDANE (Holding Fast to What is Good: A Reply to Adriaan T. Peperzak, in "American Catholic Philosophical Quarterly», 73, 1999, pp. 497-503) e A.T. PePERZAK (Personal-Impersonal? A Rejoinder to John Haldane, in "American Catholic Philosophical Quarterly», 73, 1999, pp. 503-4). Certamente, non si può tacere che permane una sorta di clima di sospetto e di "conflitto ermeneutico", tra filosofi tomisti "continentali" e "analitici", che li rende sovente ciechi e sordi in merito alla fecondità delle dottrine tomiste avanzate ora dagli uni ora dagli altri: cfr. G. VeNTIMIGLIA (To be o esse?, cit., pp. 29-74), il quale si propone di dimostrare l'infondatezza delle reciproche diffidenze in relazione alla cruciale dottrina tomista dell'essere come actus essendi.

${ }^{49} \mathrm{Cfr}$. F. KERR, The Varieties of Interpreting Aquinas, in ID., ed., Contemplating Aquinas. On the Varieties of Interpretation, University of Notre Dame Press, Notre Dame 2006, pp. 27-40: «Thomas is admitted by von Balthasar to be a "transitional figure": "Whoever does not realise how Thomas was open both to the past and to the future will misunderstand his position in the history of human thought" [H.U. VON BALTHASAR, The Theology of Karl Barth: Exposition and Interpretation, Ignatius, San Francisco1992, p. 262]. Yet, if de Lubac encouraged us to read Thomas as the inheritor of the patristic conception of natural desire for God, von Balthasar (here at least) preferred us to read Thomas more in terms of what was to come [...]. In other words, for von Balthasar here, Thomas's options already opened him to the modern account in terms of theistic proofs, natural law, and so forth. Von Balthasar's discussion of Thomas's options turns on the argument that Thomas's predominantly philosophical methodology prevents him from doing Christian theology properly» (spec. p. 32).
} 
renda giustizia alle intenzioni dei suoi promotori - in particolare Haldane - , il quale invoca al contrario una sorta di "complementarietà nella diversità" tra i due approcci a Tommaso, quello analitico e quello tradizionale-continentale (purché non si intenda, con quest'ultimo, un larvato cedimento al postmodernismo di Jacques Derrida, Michel Foucault, et al. $)^{50}$.

Laddove, infatti, la divergenza fra i due approcci si dimostri più profonda, rispetto ad una mera "differenza di stile", ciascuno di essi potrà mettere in comune ciò di cui l'altro è più carente: per esempio, le indagini analitiche sul rapporto tra significato e referente, sull'epistemologia o sulla logica, ecc., oppure, all'inverso, lo studio della psicologia morale, della conoscenza naturale di Dio, dell'ontologia, ecc.

L'adagio latino: simul stabunt simul cadent, potrebbe descrivere efficacemente la comune "aspirazione alla verità", che - a giudizio di Haldane, Maclntyre, et al. inabita sia il tomismo analitico sia quello tradizionale-continentale, pur nella pluralità delle loro espressioni.

\section{Conclusione}

Da quanto esposto sin qui, emergono alcuni argomenti salienti che giudico fondamentali per comprendere I'“habitus teologico" di Maclntyre, a cominciare dal fatto che «una prima e vitale conseguenza dell'etica delle virtù, concepita in chiave aristotelico[-tomista], è l'obbligo di rimanere aperti riguardo alla possibilità dell'esistenza di un essere trascendente ${ }^{51}$, e, dunque, parimenti, riguardo alla possibilità di una rivelazione divina: è pertanto inconcepibile che si possa essere neutrali o impassibili dinanzi a simili "evenienze" - indagate in maniera precipua dalla teologia ed è altresì evidente che sia il filosofo morale sia il filosofo della religione debbano conoscere e valutare le implicazioni teologiche, intimamente congiunte al proprio oggetto di studio, che essi sono chiamati a indagare dal punto di vista formale della

\footnotetext{
${ }^{50}$ Cfr. J. Haldane, Faithful Reason: Essays Catholic and Philosophical, Routledge, London-New York, 2004, p. xi.

${ }^{51}$ D. NIKULIN, An Interview with Alasdair Maclntyre, in «Deutsche Zeitschrift für Philosophie», 44 (1996), pp. 671-83 (spec. p. 677, corsivo aggiunto).
} 
riflessione filosofica, senza dare adito, così facendo, ad alcun illecito sconfinamento o arbitrio disciplinare. Si comprende allora la necessità, sovente disattesa, di dedicare adeguato spazio allo studio sinottico delle opere di teologia filosofica di Maclntyre, accanto a quelle più propriamente etiche: le prime costituiscono, infatti, il fisiologico complemento delle seconde, anziché un'appendice minore, e tutto sommato trascurabile, dell'etica delle virtù.

Secondo: vero è che la vicenda storica del cristianesimo muove da "eventiracconti fondatori" unici e irripetibili, cioè da quell'inestricabile insieme di esperienzeracconti di e su Gesù in cui si compie la rivelazione divina. Tuttavia, lungo l'arco dei secoli, il pensiero teologico, le credenze e la prassi di vita da essi scaturiti hanno dovuto attraversare crisi epistemologiche successive - quale fu ad esempio la "prova greca" ${ }^{52}$, ovvero l'essere giudicati in base ai correnti criteri di razionalità filosofica presenti all'epoca delle origini del cristianesimo - per consolidarsi infine, dopo innumerevoli shocks, in quella che tuttora può essere considerata una sorta di mainstream, vale a dire la tradizione aristotelico-tomista. Ciò premesso, è possibile ipotizzare, in sintonia con il pensiero di Maclntyre, che la medesima tradizione - rinvigorita dall'apporto di un inatteso affluente: il tomismo analitico e corroborata da tante sfide affrontate sino ad oggi - sia meglio posizionata al fine di poter affrontare le attuali criticità che investono il teismo cristiano, rispetto ad altre pur legittime correnti di pensiero affiorate all'interno della riflessione filosofica e teologica del XX secolo: la teologia della secolarizzazione, la teologia narrativa, la radical orthodoxy, ecc.

Terzo: l'interpretazione della Rowland - che enfatizza il concetto chiave di "tradizione narrativa" (narrative tradition) - sembra al contrario rievocare la consuetudine da cui siamo partiti: quella di attribuire al filosofo scozzese una

\footnotetext{
${ }^{52}$ Cfr. R.L. WILKEN, The Spirit of Early Christian Thought: Seeking the Face of God, Yale U.P., New HavenLondon 2003 (trad. it., Alla ricerca del volto di Dio: la nascita del pensiero cristiano, Vita e Pensiero, Milano 2006, p. 13).
} 
predilezione elettiva nei confronti della teologia narrativa, in linea con il pensiero di Frei, Hauerwas, Lindbeck, et al. ${ }^{53}$

Sebbene Maclntyre non abbia espresso alcun esplicito giudizio in merito alla teologia narrativa ${ }^{54}$, né tanto meno alla radical orthodoxy, credo sia lecito affermare che qualsiasi tradizione cristiana non possa in sé e per sé qualificarsi come narrativa, poiché esige costantemente di incarnarsi nella prassi di vita di una comunità di ricerca morale, filosofica e teologica.

Maclntyre è certamente consapevole del ruolo che la narrazione può svolgere nella formazione dell'identità personale: nel caso del cristianesimo, tale ruolo è rafforzato dal primato religioso dell'ascolto rivolto agli eventi-racconti fondatori che rivelano Dio all'umanità (fides ex auditu). Tuttavia, sono altresì convinto che - in accordo con la lezione di alcuni teologi di formazione neotomista: Lafont, et al. - egli riconosca l'ineludibile compito di dover ricercare un radicamento ontologico al di là dell'evento-parola e della forma letteraria in cui si sostanzia la narrazione biblica (così come qualunque altra specie di narrazione umana). In definitiva, infatti, racconti fondatori, prassi di vita ed eventi, che attuano e trasmettono una tradizione, possono

${ }^{53}$ Cfr. T. Rowland, Culture and the Thomist Tradition after Vatican II, cit., p. 123: "The significance of this critical concept of a "narrative tradition" should thus not be underestimated in any evaluation of Macintyre's contribution to the recent and future development of the Thomist tradition». Al riguardo, cfr. anche C.J. THOMPSON, Benedict, Thomas, or Augustine? The Character of Maclntyre's Narrative, in "The Thomist», 59 (1995), pp. 379-407.

${ }^{54}$ Ciononostante, in un recente colloquio con L. KAVANAGH (Interview: Alasdair Maclntyre, University of Notre Dame, in "Expositions», 6, 2012, pp. 1-8), Maclntyre ha così risposto alle domande dell'intervistatore: «Kavanagh: Stanley Hauerwas, for example, has suggested that you draw too sharp a distinction between [theology and philosophy; cfr. ID., The State of The University: Academic Knowledges and the Knowledge of God, Blackwell, Malden 2007, pp. 26-7]. Similarly, John Milbank has argued that, without determinate theological commitments, philosophy risks remaining bound to the conceptual frameworks of secular modernity [cfr. ID., Theology and Social Theory, cit., pp. 327-81]. How might you respond to these readers that, perhaps, you are not theological enough? Maclntyre: Philosophy, like carpentry and accountancy, is a secular trade. The standards by which its arguments and its enquiries are evaluated are those of natural reason [...]. It is in fact theology that presupposes commitment to certain philosophical positions, something recognized by the First Vatican Council in its teaching that God's existence and the natural law that he ordains for human beings, independently of his self-revelation, can be known by the light of reason [...]. The task that all contemporary theistic philosophers have is that of keeping open the question of God's existence in a culture that is often less secularized than its intelligentsias take it to be, so that modernity becomes self-questioning and self-doubting. To achieve this they have to engage in the contemporary philosophical conversation on its own terms, something insufficiently recognized by such theologians as Hauerwas and Milbank» (spec. pp. 3-4). 
essere adeguatamente compresi e riconosciuti autentici, soltanto se si salvaguarda la loro tensione verso un effettivo radicamento ontologico: e non vi è dubbio, ancora una volta, che - a giudizio di Maclntyre - tale anelito possa tutt'oggi trovare migliore appagamento nella tradizione aristotelico-tomista e nel principio analogico dell'ipsum esse subsistens ${ }^{55}$, che non nel "Dio senza essere" di Marion o nell'attesa dell' "ultimo Dio" heideggeriano ${ }^{56}$

\section{Referenze}

T. D. D'ANDREA, Tradition, Rationality, and Virtue: The Thought of Alasdair Maclntyre, Ashgate, Aldershot 2006.

A. MacINTYRE, The Debate about God, in A. Maclntyre - P. Ricoeur, The Religious Significance of Atheism, Columbia U.P., New York 1969, pp. 1-55.

A. MacINTYRE, God, Philosophy, Universities, Rowman \& Littlefield, Lanham 2009.

J. MILBANK, Theology and Social Theory: Beyond Secular Reason, Blackwell, Malden 1990.

G. LAFONT, Dieu, le temps et l'être, Éditions du Cerf, Paris 1986.

C. LEONARDI, Alasdair Maclntyre. Sul crocevia tra etica, filosofia della religione $e$ teologia, Aracne, Roma 2013.

T. ROWLAND, Culture and the Thomist Tradition after Vatican II, Routledge, London-New York 2003.

Docente di Logica e Gnoseologia Istituto Superiore di Scienze Religiose «Beato Gregorio X» (Arezzo) collegato alla Facoltà Teologica dell'Italia Centrale (Firenze) E-mail: leonardi@issrarezzo.com

\footnotetext{
${ }^{55}$ Cfr. A. MAclntYRe, God, Philosophy, Universities, Rowman \& Littlefield, Lanham 2009, pp. 73-86.

${ }^{56}$ Cfr. J.-L. MARION, Dieu sans l'être, Fayard, Paris 1982 (trad. it., Dio senza essere, Jaca Book, Milano 2008 ${ }^{2}$ ); E. ForCellino, L'ethos dell'altro inizio: appunti sulla figura dell'ultimo Dio nei "Contributi alla filosofia (dell'evento)" di Heidegger, in "Etica \& Politica/Ethics \& Politics», 11 (2009), pp. 69-91; T.S. MEl, Heidegger and the Appropriation of Metaphysics, in «The Heythrop Journal», 50 (2009), pp. 257-70, ecc.
} 